\title{
Cartan Subalgebra Approach to Efficient Measurements of Quantum Observables
}

\author{
Tzu-Ching Yen ${ }^{1}{ }^{1}$ and Artur F. Izmaylov $\circledast^{2,1, *}$ \\ ${ }^{1}$ Chemical Physics Theory Group, Department of Chemistry, University of Toronto, Toronto, Ontario M5S 3H6, \\ Canada \\ ${ }^{2}$ Department of Physical and Environmental Sciences, University of Toronto Scarborough, Toronto, Ontario MIC \\ 1A4, Canada
}

(Received 20 July 2020; revised 22 September 2021; accepted 29 September 2021; published 29 October 2021)

\begin{abstract}
An arbitrary operator corresponding to a physical observable cannot be measured in a single measurement on currently available quantum hardware. To obtain the expectation value of the observable, one needs to partition its operator into measurable fragments. However, the observable and its fragments generally do not share any eigenstates and thus the number of measurements needed to obtain the expectation value of the observable can grow rapidly even when the wave function prepared is close to an eigenstate of the observable. We provide a unified Lie-algebraic framework for developing efficient measurement schemes for quantum observables. It is based on two elements: (1) embedding the observable operator in a Lie algebra and (2) transforming Lie-algebra elements into those of a Cartan subalgebra (CSA) using unitary operators. The CSA plays the central role because all its elements are mutually commutative and thus can be measured simultaneously. We illustrate the framework on measuring expectation values of Hamiltonians appearing in the variational quantum eigensolver approach to quantum chemistry. The CSA approach puts many recently proposed methods for the measurement optimization within a single framework and allows one not only to reduce the number of measurable fragments but also the total number of measurements.
\end{abstract}

DOI: 10.1103/PRXQuantum.2.040320

\section{INTRODUCTION}

In digital quantum computing, one prepares a wave function of the simulated quantum system and any property of interest needs to be physically measured to obtain estimates that constitute the result of the computation. One popular example of this approach is the variational quantum eigensolver (VQE) [1], which is used to solve an eigenvalue problem for the Hamiltonian of interest. Note that in contrast to analog quantum computing, $[2,3]$ the Hamiltonian of interest is not encoded in VQE but, rather, its expectation value is measured. The main problem of this measurement setup is that the entire Hamiltonian cannot be measured in a single measurement. This makes efficient partitioning of operators into measurable components one of the most important problems of digital quantum computing.

Any quantum observable is represented by an operator in some mathematical form. To obtain the expectation

\footnotetext{
*artur.izmaylov@utoronto.ca
}

Published by the American Physical Society under the terms of the Creative Commons Attribution 4.0 International license. Further distribution of this work must maintain attribution to the author(s) and the published article's title, journal citation, and DOI. value of this operator in digital quantum computing, one needs to prepare the quantum system in a particular state corresponding to a wave function in the Hilbert space of $N$ qubits $(|\Psi\rangle)$ and to represent the operator of interest $\hat{O}$ in that Hilbert space:

$$
\hat{O}=\sum_{n} c_{n} \hat{P}_{n}, \hat{P}_{n}=\otimes_{k=1}^{N} \hat{\sigma}_{k}
$$

where the $c_{n}$ are numerical coefficients and the $\hat{P}_{n}$ are tensor products of single-qubit operators $\hat{\sigma}_{k}$, which are either Pauli-spin operators $\hat{x}_{k}, \hat{y}_{k}, \hat{z}_{k}$ or the identity $\hat{1}_{k}$.

Digital quantum computers can measure only singlequbit polarization along the $z$ axis. This allows one to measure straightforwardly only $2^{N}-1$ operators for $N$ qubits: $\hat{z}_{i}$ and all possible products $\hat{z}_{i} \otimes \hat{z}_{j} \cdots \otimes \hat{z}_{k}$. An arbitrary operator $\hat{O}$ can be expressed as a linear combination of such products only after applying some multiqubit unitary transformation $\hat{U}$ :

$$
\hat{O}=\hat{U}^{\dagger}\left[\sum_{i} a_{i} \hat{z}_{i}+\sum_{i j} b_{i j} \hat{z}_{i} \hat{z}_{j}+\cdots\right] \hat{U},
$$


(a)

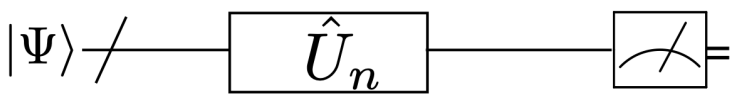

(b)

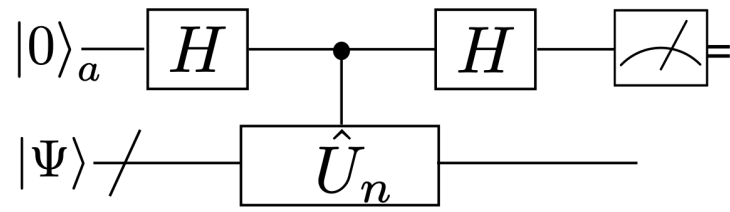

(c)

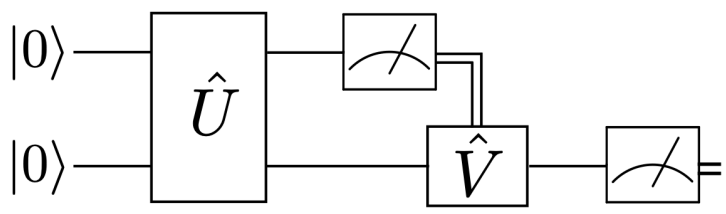

FIG. 1. Various measurement schemes involving unitary transformations: (a) measuring $\left\langle\Psi\left|\hat{U}_{n}^{\dagger} \hat{z}_{k} \hat{U}_{n}\right| \Psi\right\rangle$; (b) measuring $\operatorname{Re}\left\langle\Psi\left|\hat{U}_{n}\right| \Psi\right\rangle$ as $\pm\left(1-2 p_{ \pm 1}\right)$, where $p_{ \pm 1}$ is the probability of obtaining \pm 1 in the auxiliary qubit measurement (here, $H$ is the Hadamard gate); (c) a two-qubit example of a feed-forward scheme, where a result of the first qubit measurement defines application of the $\hat{V}$ transformation for the second qubit.

where the $a_{i}, b_{i j}, \ldots$ are some constants. The problem of obtaining $\hat{U}$ is equivalent to solving the eigenvalue problem for $\hat{O}$ and thus is hard to solve in general. However, one can take advantage of additivity of the $\hat{O}$ expectation value with respect to the $\hat{O}$ components. A feasible alternative to representation in Eq. (2) is to partition $\hat{O}$ into fragments $\hat{O}=\sum_{n} \hat{O}_{n}$, where each $\hat{O}_{n}$ can be written as Eq. (2) and has a corresponding "diagonalizing" $\hat{U}_{n}$. This raises a question: how does one select such fragments $\hat{O}_{n}$ ? Two main requirements for these fragments are that not a large number of them is required to represent the whole $\hat{O}$, and that $\hat{U}_{n}$ is not difficult to obtain using a classical computer. Having these fragments allows one to measure the expectation value of $\hat{O}$ on a wave function $|\Psi\rangle$ as in Fig. 1(a). The overall efficiency of the $\hat{O}$ measurement depends on how many measurements each fragment requires to obtain accurate estimates of its expectation value. This consideration makes fragment variances, $\left\langle\hat{O}_{n}^{2}\right\rangle-\left\langle\hat{O}_{n}\right\rangle^{2}$, the key quantities for efficient measurement.

There are multiple ways to choose $\hat{O}_{n}$ fragments considering the two conditions. For example, one can try to find exactly solvable models within $\hat{O}$ as $\hat{O}_{n}$ fragments, but some models are not straightforward to find within $\hat{O}$ and their sizes may be small. Also, the sum of two exactly solvable models may not necessarily be another exactly solvable model, which prevents combining such models into larger fragments. One of the most productive approaches so far has been to try to increase the size of $\hat{O}_{n}$ by collecting all terms of $\hat{O}$ that mutually commute. The condition of mutual commutativity allows all commuting operators in each $\hat{O}_{n}$ to be simultaneously diagonalizable or to have one unitary operator, $\hat{U}_{n}$, transforming all of them into all $\hat{z}_{i}$ form as in Eq. (2). This idea has been applied to the electronic Hamiltonian in both qubit [4-9] and fermionic $[10,11]$ operator forms. It has been found that working in the fermionic representation provides superior results compared to those obtained in the qubit case $[10,11]$. Even though all these methods stem from the same idea of finding commuting terms, there has been no general framework that would encompass all of the mentioned methods developed so far. This work provides such a framework, with solid foundations in theory of Lie algebras. Moreover, the introduced framework allows us to propose an extension of previous approaches that results in lower numbers of measurements required to accurately obtain an expectation value.

As alternatives to the simple measurement scheme presented in Fig. 1(a), a few more sophisticated schemes have been put forward for efficient measurement [see Figs. 1(b) and 1(c)]. The scheme in Fig. 1(b) exploits partitioning of the operator into a linear combination of unitaries that can be measured indirectly via measuring the orientation of an auxiliary qubit (the Hadamard test) [12]. This scheme requires efficient partitioning of the operator into unitary components and their implementation in controlled form. Such controlled unitaries are generally more expensive in terms of two-qubit gates than their noncontrolled counterparts. The scheme in Fig. 1(c) uses results of previous measurements to adjust the next measurements - this is a so-called feed-forward scheme. Recently, it has become available not only in laboratories [13] but also in an actual quantum computer produced by Honeywell [14]. The efficiency of this scheme depends on partitioning of the operator into measurable fragments. Even though the definition of such fragments have been introduced [15], there is still no systematic procedure to do the optimal partitioning of an arbitrary operator into such fragments.

Yet another class of measurement techniques that has appeared recently is that of methods based on shadow tomography [16-18]. It is based on stochastic sampling of unitary rotations $\hat{U}_{n}$ for optimal measurement of an operator set $\hat{O}_{n}$. Even though this technique has recently been used for measurement of electronic Hamiltonians [19-21], its careful comparison with the deterministic partitions developed here goes beyond the scope of the current work and will be reported elsewhere [22].

The rest of the paper is organized as follows. Section II A shows how a quantum observable operator $\hat{O}$ can be expressed using elements of different Lie algebras. Section II B details the role of the CSA for the measurement of $\hat{O}$ and CSA decomposition. Section III illustrates how 
application of the CSA decomposition to the fermionic and qubit algebras connects several existing approaches and Sec. IV proposes schemes improving CSA decompositions in the number of measurements. Section V assesses the new approach and several existing methods on a set of molecular systems $\left(\mathrm{H}_{2}, \mathrm{LiH}, \mathrm{BeH}_{2}, \mathrm{H}_{2} \mathrm{O}, \mathrm{NH}_{3}\right.$, and $\mathrm{N}_{2}$ ). Section VI summarizes the main results and provides concluding remarks.

\section{THEORY}

\section{A. Operator embeddings}

Any operator in quantum mechanics can be written as a polynomial expression of some elementary operators $\left\{\hat{A}_{k}\right\}$ :

$$
\hat{O}=\sum_{k} c_{k} \hat{A}_{k}+\sum_{k k^{\prime}} d_{k k^{\prime}} \hat{A}_{k} \hat{A}_{k^{\prime}}+\cdots
$$

where $\left\{\hat{A}_{k}\right\}$ form a Lie algebra $\mathcal{A}$ with respect to the commutation operation

$$
\left[\hat{A}_{i}, \hat{A}_{j}\right]=\sum_{k} \xi_{i j}^{(k)} \hat{A}_{k}, \hat{A}_{i}, \hat{A}_{j}, \hat{A}_{k} \in \mathcal{A}
$$

Here, the $\xi_{i j}^{(k)}$ are so-called structural constants from the number field $\mathbb{K}$ [23]. Note that products such as $\hat{A}_{k} \hat{A}_{k^{\prime}}$ and higher powers of $\mathcal{A}$ elements do not generally belong to the Lie algebra $\mathcal{A}$; instead, they are part of a universal enveloping algebra (UEA), $\mathcal{E}_{\mathcal{A}}$, which is built as a direct sum of tensor powers of the Lie algebra

$$
\mathcal{E}_{\mathcal{A}}=\mathbb{K} \oplus \mathcal{A} \oplus(\mathcal{A} \otimes \mathcal{A}) \oplus \cdots,
$$

where the Lie bracket operation is equivalent to the commutator. Thus, any operator in quantum mechanics is an element of some UEA.

Any operator for a physical observable is self-adjoint (i.e., Hermitian), which allows us to only consider compact Lie algebras (i.e., all generators are Hermitian) for expressing such operators. Any compact Lie algebra can be expressed as a direct sum of Abelian and semisimple Lie algebras [24]. For considering the measurement problem within compact Lie algebras, it is convenient to introduce the notion of the Cartan subalgebra $\mathcal{C} \subset \mathcal{A}$, which in this case, is a maximal Abelian subalgebra [25]. We denote elements of $\mathcal{C}$ as $\hat{C}_{k}$. The UEA constructed from $\mathcal{C}, \mathcal{E}_{\mathcal{C}}$, is Abelian as well. Thus, in principle, all elements of $\mathcal{E}_{\mathcal{C}}$ can be measured simultaneously. In practice, there is need for unitary transformations that transform $\mathcal{E}_{\mathcal{C}}$ elements of a particular CSA into those of all $\hat{z}$ qubit operators. For all algebras discussed in this work, these transformations involve only standard fermion-qubit mappings.

One operator can be written as an element of different UEAs, where different UEAs are built from different
Lie algebras. We refer to expressions of the same operator using different Lie algebras as different embeddings. Various embeddings of the electronic Hamiltonian are illustrated in Appendix A.

\section{B. Cartan subalgebra approach}

We illustrate how elements of $\mathcal{E}_{\mathcal{C}}$ can be used for measuring operator $\hat{O}$ that can be written using elements of UEA of a Lie algebra $\mathcal{A}$ up to quadratic terms, $\hat{A}_{k} \hat{A}_{k^{\prime}}$ in Eq. (3). This case can be easily generalized to operators that contain higher powers of $\hat{A}_{k}$. Also, we generally assume that $\hat{A}_{k} \hat{A}_{k^{\prime}}$ do not belong to the Lie algebra $\mathcal{A}$. To use elements of $\mathcal{E}_{\mathcal{C}}$, we need to find a minimum number of unitary transformations that allow us to present the operator of interest as

$$
\hat{O}=\sum_{\alpha=1}^{M} \hat{U}_{\alpha}^{\dagger}\left[\sum_{l}^{|\mathcal{C}|} \lambda_{l}^{(1, \alpha)} \hat{C}_{l}+\sum_{l l^{\prime}}^{|\mathcal{C}|} \lambda_{l l^{\prime}}^{(2, \alpha)} \hat{C}_{l} \hat{C}_{l^{\prime}}\right] \hat{U}_{\alpha}
$$

where $\hat{C}_{l} \in \mathcal{C},|\mathcal{C}|$ is the CSA size, and $\lambda_{l}^{(1, \alpha)}$ and $\lambda_{l l^{\prime}}^{(2, \alpha)}$ are some tensors.

What are the possible candidates for $\hat{U}_{\alpha}$ ? Clearly, they should depend on the $\hat{A}_{k}$ and they should not create complicated expressions when acting on CSA elements. In this work, we consider two constructions of $\hat{U}_{\alpha}$ transformations but generally one can search for other ways to construct $\hat{U}_{\alpha}$ transformations as functions of the $\hat{A}_{k}$. The main guiding principle in this search can be a requirement that $\hat{U}_{\alpha}$ transformation of any element of $\mathcal{E}_{\mathcal{C}}$ produces a low-degree polynomial number of terms from $\mathcal{E}$.

\section{Lie-group unitaries}

The first approach for $\hat{U}_{\alpha}$ transformations is to take the elements of the corresponding Lie group:

$$
\hat{U}_{\alpha}=\exp \left[i \sum_{k}^{|\mathcal{A}|} \hat{A}_{k} \theta_{k}^{(\alpha)}\right]
$$

where $\theta_{k}^{(\alpha)} \in \mathbb{R},|\mathcal{A}|$ is the Lie-algebra size and the $\hat{A}_{k}$ are assumed to be Hermitian. Due to the closure in $\mathcal{A}$, we have

$$
\hat{U}_{\alpha}^{\dagger}\left[\sum_{k}^{|\mathcal{A}|} c_{k} \hat{A}_{k}\right] \hat{U}_{\alpha}=\sum_{k}^{|\mathcal{A}|} c_{k}^{(\alpha)} \hat{A}_{k},
$$

where the $c_{k}^{(\alpha)}$ are some constants. Essentially, the Liegroup elements $\hat{U}_{\alpha}$ provide a set of automorphisms for the corresponding Lie algebra. Moreover, according to the maximal tori theorem for compact groups (all algebras involved in the Hamiltonian embeddings correspond to 
compact groups) [26], it is guaranteed that there exists a choice of $\theta_{k}^{(\alpha)}=\theta_{k}$ in $\hat{U}_{\alpha}=\hat{U}\left(\theta_{k}\right)$ satisfying

$$
\hat{U}\left[\sum_{k}^{|\mathcal{A}|} c_{k} \hat{A}_{k}\right] \hat{U}^{\dagger}=\sum_{l}^{|\mathcal{C}|} \lambda_{l}^{(1)} \hat{C}_{l}
$$

for any values of $c_{k}$. The maximal tori theorem provides a basis for finding a single $\hat{U}$ that transforms the linear part of $\hat{O}$ into a linear combination of the CSA terms. Amplitudes $\boldsymbol{\theta}=\left\{\theta_{k}\right\}$ for this $\hat{U}$ can be found numerically by solving the system of equations

$$
c_{k}=\sum_{l}^{|\mathcal{C}|} \lambda_{l}^{(1)} c_{k}^{(l)}(\boldsymbol{\theta})
$$

where the $c_{k}^{(l)}(\boldsymbol{\theta})$ are some functions the explicit form of which depends on the Lie algebra. Thus, in what follows, we can focus on representation of the quadratic part of $\hat{O}$, $\hat{O}^{(2)}$.

Since the $\hat{U}_{\alpha}$ operators in Eq. (7) do not change the power of the $\hat{A}_{k}$ after transformation (8), one can find $\theta_{l}^{(\alpha)}$ and $\lambda_{l l^{\prime}}^{(2, \alpha)}$ by minimization of the difference between the quadratic parts of Eqs. (3) and (6). To facilitate the process, it is useful to introduce the appropriate basis in the UEA. Such bases are given by the Poincaré-Birkhoff-Witt theorem [27]:

$$
\begin{gathered}
\text { basis } 1:\left\{1, \hat{A}_{k_{1}}, \hat{A}_{k_{1}} \hat{A}_{k_{2}}\right\}, \\
\text { basis } 2:\left\{1, \hat{A}_{k_{1}},\left(\hat{A}_{k_{1}} \hat{A}_{k_{2}}+\hat{A}_{k_{2}} \hat{A}_{k_{1}}\right) / 2\right\},
\end{gathered}
$$

where $k_{2} \leq k_{1}=1, \ldots,|\mathcal{A}|$. Note that these bases can be continued to the higher polynomial functions of the $\hat{A}_{k}$ but we do not need them beyond the quadratic terms.

Both representations of $\hat{O}$ [Eqs. (3) and (6)] can be transformed into a linear combination of the basis elements. Symmetric basis 2 is somewhat simpler to work with and thus we use it here, denoting $\left\{\hat{A}_{k_{1}} \hat{A}_{k_{2}}\right\}_{S}=\left(\hat{A}_{k_{1}} \hat{A}_{k_{2}}+\right.$ $\left.\hat{A}_{k_{2}} \hat{A}_{k_{1}}\right) / 2$. Assuming the Hermiticity of $d_{k k^{\prime}}$, the quadratic part of Eq. (3) transforms to

$$
\hat{O}^{(2)}=\sum_{k \geq k^{\prime}}^{|\mathcal{A}|} d_{k k^{\prime}}\left(2-\delta_{k k^{\prime}}\right)\left\{\hat{A}_{k} \hat{A}_{k^{\prime}}\right\}_{S}
$$

The $\hat{O}^{(2)}$ part of Eq. (6) can be written as

$$
\hat{O}^{(2)}=\sum_{\alpha=1}^{M} \sum_{l l^{\prime}}^{|\mathcal{C}|} \lambda_{l l^{\prime}}^{(2, \alpha)}\left[\hat{U}_{\alpha}^{\dagger} \hat{C}_{l} \hat{U}_{\alpha}\right]\left[\hat{U}_{\alpha}^{\dagger} \hat{C}_{l^{\prime}} \hat{U}_{\alpha}\right] .
$$

Applying the unitary transformation to CSA elements,

$$
\hat{U}_{\alpha}^{\dagger} \hat{C}_{l} \hat{U}_{\alpha}=\sum_{k}^{|\mathcal{A}|} c_{k}^{(l)}\left(\boldsymbol{\theta}^{\alpha}\right) \hat{A}_{k},
$$

leads to

$$
\begin{aligned}
\hat{O}^{(2)}= & \sum_{k \geq k^{\prime}}^{|\mathcal{A}|}\left[\sum_{\alpha=1}^{M} \sum_{l l^{\prime}}^{|\mathcal{C}|} \lambda_{l l^{\prime}}^{(2, \alpha)} c_{k}^{(l)}\left(\boldsymbol{\theta}^{\alpha}\right) c_{k^{\prime}}^{\left(l^{\prime}\right)}\left(\boldsymbol{\theta}^{\alpha}\right)\right] \\
& \left(2-\delta_{k k^{\prime}}\right)\left\{\hat{A}_{k} \hat{A}_{k^{\prime}}\right\}_{S} .
\end{aligned}
$$

Term-wise comparison of Eqs. (13) and (16) gives equations in $\boldsymbol{\lambda}^{(2, \alpha)}$ and $\boldsymbol{\theta}^{(\alpha)}$ :

$$
d_{k k^{\prime}}=\sum_{\alpha=1}^{M} \sum_{l l^{\prime}}^{|\mathcal{C}|} \lambda_{l l^{\prime}}^{(2, \alpha)} c_{k}^{(l)}\left(\boldsymbol{\theta}^{(\alpha)}\right) c_{k^{\prime}}^{\left(l^{\prime}\right)}\left(\boldsymbol{\theta}^{(\alpha)}\right),
$$

where the only functions to derive are $c_{k}^{(l)}\left(\boldsymbol{\theta}^{\alpha}\right)$.

This consideration can be extended to higher powers of Lie-algebra elements beyond quadratic. However, for such extensions, the number of equations will grow exponentially with the algebraic degree and become computationally overwhelming.

\section{Number of terms conserving unitaries}

An alternative that is more efficient for cases with higher powers of the $\hat{A}_{k}$ is to use unitary operators $\hat{U}_{\alpha}$ that conserve the number of terms in the transformation:

$$
\hat{U}_{\alpha}^{\dagger}\left(\prod_{i} \hat{C}_{k_{i}}\right) \hat{U}_{\alpha}=\prod_{j} \hat{A}_{k_{j}}
$$

The functional form of $\hat{U}_{\alpha}=\hat{U}_{\alpha}\left(\left\{\hat{A}_{k}\right\}\right)$ depends on associative algebraic properties of the UEA used and cannot be given explicitly for a general UEA. For example, in the qubit embedding, every element of the Lie algebra is involutory, $\hat{A}_{k} \hat{A}_{k}=\hat{1}$, which leads to the $\hat{U}_{\alpha}$ operators being elements of the Clifford group.

Since all elements of $\mathcal{E}_{\mathcal{C}}$ commute among themselves, the resulting products of $\hat{A}_{k_{j}}$ in Eq. (18) are also commutative. Thus, in practice, to find a linear combination of operator terms that can be treated by a single $\hat{U}_{\alpha}$ operator, one needs to group all mutually commuting terms. Generally, the commutation relation between operator terms can be represented by a graph the edges of which connect commuting terms represented by vertices. Partitioning of the $\hat{O}$ expression into mutually commuting groups can be done in various ways but finding the optimal partitioning into a minimum number of such groups is a standard NP-hard problem in graph theory, the minimum-clique-cover problem. There are many heuristic polynomial algorithms to solve this problem [4]. 


\section{APPLICATIONS}

Here, we illustrate how novel algorithms and several previously developed methods can be derived from the CSA framework applied to the electronic Hamiltonian $\hat{H}_{e}$ as an example of a many-body operator $\hat{O}$. Fermionic and qubit embeddings are used to illustrate the CSA decomposition of $\hat{H}_{e}$. As shown in Appendix A, the fermionic embeddings contain low powers of Lie-algebra elements and thus one can expect more benefits from using the Liegroup unitaries [Eq. (7)], while the qubit embedding has the involutory associative multiplication property for the algebra elements and thus benefits more from the number of terms conserving unitaries [Eq. (18)].

\section{A. Fermionic algebras}

To apply the Lie-group unitaries [Eq. (7)], one needs operator embeddings involving compact Lie algebras. Even though we use generators of the noncompact $\mathfrak{g l}(N)$ algebra, denoted $\hat{E}_{p}^{q}$, this does not create any problem, because in what follows the $\hat{E}_{p}^{q}$ always appear as linear combinations $i\left(\hat{E}_{p}^{q}-\hat{E}_{q}^{p}\right)$ and $\left(\hat{E}_{p}^{q}+\hat{E}_{q}^{p}\right)$, which are generators of the compact Lie algebra $\mathfrak{u}(N)$. The origin of this compactness is the Hermiticity of the system Hamiltonian, which allows us to rewrite the Hamiltonian in $\mathfrak{u}(N)$ generators. The CSAs of $\mathfrak{g l}(N)$ and $\mathfrak{u}(N)$ are the same: $N$ elements $\hat{E}_{p}^{p}$.

The $\hat{E}_{p}^{q}$ are isomorphic to real $N$ by $N$ matrices $\left(E_{q}^{p}\right)_{m n}=$ $\delta_{m p} \delta_{n q}$ and there are $N^{2}$ such elements. This faithful representation of $\hat{E}_{p}^{q}$ makes the search for $\hat{U}_{\alpha}$ in Eq. (9) equivalent to a simple diagonalization of the Hermitian matrix, $h_{p q}$.

Unfortunately, the two-electron part of the fermionic Hamiltonian cannot be treated as easily as the one-electron part. Products $\hat{E}_{p}^{q} \hat{E}_{r}^{s}$ do not form a Lie algebra and thus their representation as tensor products of pairs of $N$ by $N$ matrices do not lead to the unitary transformations that we are looking for. However, diagonalization of fourindex tensors $g_{p q, r s}$ can provide some approximation to the CSA decomposition and is discussed as a Hamiltonian factorization.

\section{Hamiltonian factorization}

Using the maximal tori theorem, one can employ the following heuristic approach to obtaining the expansion for the quadratic part $\hat{O}^{(2)}$ in products of linear combinations of $\hat{A}_{k}$ :

$$
\hat{O}^{(2)}=\sum_{\alpha=1}^{M^{\prime}}\left(\sum_{k}^{|\mathcal{A}|} c_{k}^{(\alpha)} \hat{A}_{k}\right)^{2}
$$

$$
\begin{gathered}
=\sum_{k, k^{\prime}}^{|\mathcal{A}|} \hat{A}_{k} \hat{A}_{k^{\prime}} \sum_{\alpha=1}^{M^{\prime}}\left[c_{k}^{(\alpha)} c_{k^{\prime}}^{(\alpha)}\right] \\
=\sum_{k, k^{\prime}}^{|\mathcal{A}|} \tilde{d}_{k k^{\prime}} \hat{A}_{k} \hat{A}_{k^{\prime}},
\end{gathered}
$$

where $\tilde{d}_{k k^{\prime}}$ is an analog of $d_{k k^{\prime}}$ from Eq. (3). Thus, one can decompose $d_{k k^{\prime}}$ as $\sum_{\alpha=1}^{M^{\prime}}\left[c_{k}^{(\alpha)} c_{k^{\prime}}^{(\alpha)}\right]$ and then find $\hat{U}_{\alpha}$ by applying the maximal tori theorem for each linear combination $\sum_{k} c_{k}^{(\alpha)} \hat{A}_{k}$. Yet, considering that the rank for $\lambda_{l l^{\prime}}^{(2, \alpha)}$ in Eq. (6) can be $|\mathcal{C}|$ (full rank) and that of $c_{k}^{(\alpha)} c_{k^{\prime}}^{(\alpha)}$ for each $\alpha$ in Eq. (20) is only 1, one can see that the representation of Eq. (6) can be more compact than that of Eq. (20), $M<M^{\prime}$.

A heuristic treatment of the quadratic part of $\hat{O}$ by decomposing $d_{k k^{\prime}}$ into $c_{k}^{(\alpha)} c_{k^{\prime}}^{(\alpha)}$ in Eq. (20) can be done using singular-value decomposition (SVD). Huggins et al. [10] do exactly that for the two-electron part of the fermionic Hamiltonian, where $\left\{d_{k k^{\prime}}\right\}=\left\{g_{p q, r s}\right\}$ and $\left\{\hat{A}_{k}\right\}=$ $\left\{\hat{E}_{p}^{q}\right\}$ (details are given in Appendix B).

\section{Full-rank optimization}

Extension of the factorization approach to Eq. (6) requires substitution of SVD by a more general decomposition. To arrive at Eq. (6), one can start with the symmetrized form of $\hat{H}_{e}^{(2)}$ :

$$
\hat{H}_{e}^{(2)}=\sum_{p q r s}^{N} g_{p q, r s}\left\{\hat{E}_{p}^{q} \hat{E}_{r}^{s}\right\}_{S}
$$

where $g_{p q, r s}=g_{r s, p q}$ due to permutation symmetry in the two-electron integrals. The equivalent of Eq. (6) for this Hamiltonian is

$$
\begin{aligned}
\hat{H}_{e}^{(2)} & =\sum_{\alpha=1}^{M} \hat{U}_{\alpha}^{\dagger}\left[\sum_{t u}^{N} \lambda_{t u}^{(2, \alpha)} \hat{E}_{t}^{t} \hat{E}_{u}^{u}\right] \hat{U}_{\alpha} \\
& =\sum_{\alpha=1}^{M} \sum_{t u}^{N} \lambda_{t u}^{(2, \alpha)}\left[\hat{U}_{\alpha}^{\dagger} \hat{E}_{t}^{t} \hat{U}_{\alpha}\right]\left[\hat{U}_{\alpha}^{\dagger} \hat{E}_{u}^{u} \hat{U}_{\alpha}\right],
\end{aligned}
$$

where

$$
\hat{U}_{\alpha}=\exp \left[\sum_{t>u}^{N}-i \theta_{t u}^{(\alpha)}\left(\hat{E}_{t}^{u}+\hat{E}_{u}^{t}\right)+\phi_{t u}^{(\alpha)}\left(\hat{E}_{t}^{u}-\hat{E}_{u}^{t}\right)\right] .
$$

There is a homomorphism of these unitary operators onto unitary matrices that are obtained by substituting the excitation operators $\hat{E}_{t}^{u}$ by matrices $E_{u}^{t}$, which is the faithful 
representation of algebraic generators. This homomorphism allows us to perform the operator transformations in Eq. (23),

$$
\hat{U}_{\alpha}^{\dagger} \hat{E}_{t}^{t} \hat{U}_{\alpha}=\sum_{p q} \hat{E}_{p}^{q} c_{p q}^{(t)}\left(\boldsymbol{\theta}^{(\alpha)}, \boldsymbol{\phi}^{(\alpha)}\right)
$$

by substituting the operators with corresponding matrices. This substitution gives coefficients $c_{p q}^{(t)}\left(\boldsymbol{\theta}^{(\alpha)}, \boldsymbol{\phi}^{(\alpha)}\right)$ via linear algebra. The assembly of the operator expression of Eq. (23) after the transformation of the CSA elements provides

$$
\begin{aligned}
\hat{H}_{e}^{(2)} & =\sum_{\alpha=1}^{M} \sum_{t u}^{N} \lambda_{t u}^{(2, \alpha)} c_{p q}^{(t)}\left(\boldsymbol{\theta}^{(\alpha)}, \boldsymbol{\phi}^{(\alpha)}\right) c_{r s}^{(u)}\left(\boldsymbol{\theta}^{(\alpha)}, \boldsymbol{\phi}^{(\alpha)}\right) \\
& \times\left\{\hat{E}_{p}^{q} \hat{E}_{r}^{s}\right\}_{S} .
\end{aligned}
$$

Using independence of the basis-set elements $\left\{\hat{E}_{p}^{q} \hat{E}_{r}^{s}\right\}_{S}$, the following system of algebraic equations can be written:

$$
\begin{aligned}
g_{p q, r s} & =\sum_{\alpha=1}^{M} \sum_{t u}^{N} \lambda_{t u}^{(2, \alpha)} c_{p q}^{(t)}\left(\boldsymbol{\theta}^{(\alpha)}, \boldsymbol{\phi}^{(\alpha)}\right) c_{r s}^{(u)}\left(\boldsymbol{\theta}^{(\alpha)}, \boldsymbol{\phi}^{(\alpha)}\right) \\
& =\sum_{\alpha=1}^{M} f_{p q, r s}^{(\alpha)}\left(\lambda^{(2, \alpha)}, \boldsymbol{\theta}^{(\alpha)}, \boldsymbol{\phi}^{(\alpha)}\right) .
\end{aligned}
$$

Its solution is found via gradient minimization of the norm for the vector of differences between the right- and lefthand sides using the Broyden-Fletcher-Goldfarb-Shanno (BFGS) algorithm [28].

\section{B. Qubit algebras}

There are two compact semisimple qubit algebras that can be used for the CSA decomposition of the qubit counterpart of the electronic Hamiltonian, $\hat{H}_{q}$ (Appendix A): (1) UEA of $\mathcal{S}=\oplus_{k=1}^{N} \mathfrak{s u}(2)_{k}, \mathcal{E}_{\mathcal{S}}$, or (2) $\mathfrak{s o}\left(2^{N}\right)$. One advantage of $\mathcal{E}_{\mathcal{S}}$ is a single-particle picture (elements of $\mathcal{S})$ that can define a class of computationally feasible unitaries, $\hat{U}_{\alpha}$.

\section{Single-qubit unitaries}

The Lie group constructed by exponentiating the $\mathcal{S}$ algebra consists of the following elements:

$$
\hat{U}_{\mathrm{QMF}}=\prod_{k=1}^{N} e^{i \tau_{k}\left(\bar{n}_{k}, \bar{\sigma}_{k}\right)},
$$

where $\tau_{k}$ is an amplitude, $\bar{n}_{k}$ is a unit vector on the Bloch sphere, and $\bar{\sigma}_{k}=\left(\hat{x}_{k}, \hat{y}_{k}, \hat{z}_{k}\right)$. The fragments that can be measured after $\hat{U}_{\mathrm{QMF}}$ transformations are exactly solvable within the qubit mean-field (QMF) approach [29]:

$$
\hat{H}_{\mathrm{QMF}}=\hat{U}_{\mathrm{QMF}}^{\dagger}\left[\sum_{k} a_{k} \hat{C}_{k}^{(\mathrm{Z})}\right] \hat{U}_{\mathrm{QMF}},
$$

where the $\hat{C}_{k}^{(Z)}$ are elements from the directly measurable CSA: $\left\{1, \hat{z_{i}}, \hat{z_{i}} \hat{z}_{j}, \ldots\right\}$. One-qubit rotations in the qubit space do not translate to one-electron fermionic transformations, as has been shown in Ref. [29]. Therefore, the fragments in Eq. (29) are different from those in Eq. (23). The latter are also exactly solvable but with the fermionic one-particle transformations.

Identification of fragments of Eq. (29) is not straightforward and requires a tensor decomposition of the qubit Hamiltonian, $\hat{H}_{q}$. Note that since $\hat{U}_{\mathrm{QMF}}$ transformations are not necessarily in the Clifford group [30], each of them can produce exponentially many Pauli products by transforming a single product. The number of terms in the directly measurable CSA in Eq. (29) can also be exponentially large. Thus, even though the total number of $\hat{H}_{q}$ terms is $O\left(N^{4}\right)$, a general tensor decomposition $[31,32]$ of $\hat{H}_{q}$ into fragments can have an exponential computational cost and therefore is unfeasible on a classical computer.

To avoid exponential growth of terms, one can restrict $\hat{U}_{\mathrm{QMF}}$ to a Clifford subset of transformations, thus switching from the Lie-group unitaries [Eq. (7)] to the unitaries conserving the numbers of terms [Eq. (18)]. Then, the only fragments of $\hat{H}_{q}$ that will be transformed into $\hat{C}_{k}^{(Z)}$ are the qubit-wise commuting (QWC) sets of Pauli products [4]. A practical way to find all QWC sets of Pauli products is found through mapping these problems to the minimum-clique-cover problem for a graph representing the qubit-wise commutativity relation in $\hat{H}_{q}$.

\section{Multiqubit unitaries}

An alternative consideration of $\hat{H}_{q}$ within the $\mathfrak{s o}\left(2^{N}\right)$ algebra has an advantage that the maximal tori theorem guarantees the existence of a single $\hat{U} \in \operatorname{Spin}\left(2^{N}\right)$ [33] for the transformation

$$
\hat{U} \hat{H}_{q} \hat{U}^{\dagger}=\sum_{k} a_{k} \hat{C}_{k}^{(Z)}
$$

Unfortunately, this unitary requires an exponential number of algebra elements, $\hat{U}=\exp \left[i \sum_{k} c_{k} \hat{P}_{k}\right]$. Therefore, the exponential size of $\mathfrak{s o}\left(2^{N}\right)$ prevents us from taking advantage of the maximal tori theorem. For practical purposes, we restrict $\hat{U}$ to unitaries from the Clifford group that conserve the number of terms in Eq. (18). In this case, a single $\hat{U}$ is not sufficient and $\hat{H}_{q}$ decomposition involves several 
terms:

$$
\begin{aligned}
\hat{H}_{q} & =\sum_{\alpha} \hat{H}_{q}^{(\alpha)} \\
& =\sum_{\alpha} \hat{U}_{\alpha}^{\dagger}\left[\sum_{k} a_{k}^{(\alpha)} \hat{C}_{k}^{(Z)}\right] \hat{U}_{\alpha} .
\end{aligned}
$$

To find the $\hat{U}_{\alpha}$ operators, one can use the main property of the Clifford group that any Pauli product is transformed by an element of the Clifford group to another Pauli product. This means that any fragment $\hat{H}_{q}^{(\alpha)}$ consists of commuting Pauli products, because their Clifford unitary images $\hat{C}_{k}^{(Z)}$ are also commuting. This consideration connects Eq. (31) with a partitioning method based on finding fully commuting (FC) sets, described in Ref. [6]. FC sets are also found by heuristic solutions of the minimum-clique-cover problem for a graph built for $\hat{H}_{q}$ using the commutativity relation.

\section{MEASUREMENT OPTIMIZATION}

In this section, we review the standard approach to computing the number of measurements $K$ needed to estimate the expectation value of the electronic Hamiltonian within error $\epsilon$. For a decomposition of the Hamiltonian

$$
\hat{H}=\sum_{\alpha=1}^{M} \hat{H}^{(\alpha)}
$$

where the expectation value of each fragment $\hat{H}^{(\alpha)}$ is sampled $K^{(\alpha)}$ times, the total number of measurements is $K=$ $\sum_{\alpha} K^{(\alpha)}$. As shown previously [9,34], the optimal choice of $K^{(\alpha)}$ is

$$
K^{(\alpha)}=\frac{1}{\epsilon^{2}} \sqrt{\operatorname{Var}_{\Psi}\left(\hat{H}^{(\alpha)}\right)} \sum_{\beta=1}^{M} \sqrt{\operatorname{Var}_{\Psi}\left(\hat{H}^{(\beta)}\right)},
$$

where $\epsilon$ is the desired accuracy, $|\Psi\rangle$ is the VQE trial wave function, and $\operatorname{Var}_{\Psi}\left(\hat{H}^{(\alpha)}\right)=\left\langle\Psi\left|\hat{H}^{(\alpha) 2}\right| \Psi\right\rangle-$ $\left\langle\Psi\left|\hat{H}^{(\alpha)}\right| \Psi\right\rangle^{2}$ is the operator variance. Then the total number of measurements is

$$
K=\frac{1}{\epsilon^{2}}\left(\sum_{\alpha=1}^{M} \sqrt{\operatorname{Var}_{\Psi}\left(\hat{H}^{(\alpha)}\right)}\right)^{2}
$$

where the square of the expression in the brackets can be seen as the variance of the estimators for $\langle\Psi|\hat{H}| \Psi\rangle$ originating from the decomposition in Eq. (32).

There are two main heuristics one could employ to reduce $K$. First, it is generally favorable to have an uneven distribution of $\operatorname{Var}\left(\hat{H}_{\alpha}\right)$ due to the square-root functions [9]. One can achieve this by iteratively applying full-rank optimization (FRO) with few $M$ until convergence, where at each iteration one finds the fragment that minimizes the norm of the remaining coefficients using the "greedy" approach. This approach generally results in concentrated coefficients and thus larger variances in the first few fragments. Second, one can approximate $K$ with $K_{\varphi}$ computed using wave function $|\varphi\rangle$ calculated from classical methods [e.g., Hartree-Fock (HF)] and then search for partitioning that minimizes $K_{\varphi}$. Based on these two approaches, we propose the following modifications of the FRO that reduce the total number of measurements:

(a) Greedy FRO (GFRO). This algorithm iteratively applies the FRO with $M=1$. At the $\alpha$ th iteration, the GFRO finds the $\lambda^{(2, \alpha)}, \boldsymbol{\theta}^{(\alpha)}, \boldsymbol{\phi}^{(\alpha)}$ of Eq. (27) that minimizes

$$
\left|g_{p q, r s}-\sum_{\beta=1}^{\alpha-1} f_{p q, r s}^{(\beta)}-f_{p q, r s}^{(\alpha)}\left(\lambda^{(2, \alpha)}, \boldsymbol{\theta}^{(\alpha)}, \boldsymbol{\phi}^{(\alpha)}\right)\right|^{2},
$$

where the parameters of $f_{p q, r s}^{(\beta)}$ are fixed from previous iterations.

(b) Variance-estimate greedy FRO (VGFRO). This algorithm also uses FRO with $M=1$ consecutively and fixes coefficients from previous iterations. Unlike the GFRO, the VGFRO minimizes

$$
\left|d_{p q, r s}^{(\alpha)}\right|^{2}+w \operatorname{Var}_{\varphi}\left(\hat{H}^{(\alpha)}\right)
$$

at the $\alpha$ th iteration, where

$$
\begin{aligned}
& d_{p q, r s}^{(\alpha)}=g_{p q, r s}-\sum_{\beta=1}^{\alpha} f_{p q, r s}^{(\beta)} \\
& \hat{H}^{(a)}=\sum_{p q r s}^{N} f_{p q, r s}^{(\alpha)}\left\{\hat{E}_{p}^{q} \hat{E}_{r}^{s}\right\}_{S}
\end{aligned}
$$

and $w$ is a fixed parameter. Note that one needs to choose a small enough $w$ to encourage the optimization to reduce $\left|d_{p q, r s}^{(\alpha)}\right|^{2}$ despite the second term of Eq. (36), which is minimized when $f_{p q, r s}^{(\alpha)}=0$. In practice, the variances of the first few fragments obtained from GFROs are significantly larger than those of the fragments from subsequent iterations. Thus, for computational efficiency of the VGFRO, we drop the second term of Eq. (36) after the first $\mu$ fragments. This is equivalent to performing a GFRO on the remaining $d_{p q, r s}^{(\mu)}$ tensor.

(c) Qubit-based algorithms. In qubit algebra, the greedy approach naturally leads to the sorted-insertion (SI) 
algorithm [9], which also groups the commuting Pauli products with large coefficients together in a few measurable fragments. However, further utilizing the approximated variance in SI is not straightforward, since partitioning of the qubit Hamiltonian is not guided by gradients that are used to minimize the norm and variance in a continuous fashion in the VGFRO.

\section{RESULTS}

To assess the CSA decomposition techniques in fermionic and qubit algebras, we apply them to a set of Hamiltonians previously used to demonstrate the performance of similar measurement techniques $[4,6,12]$ (Tables I and II). Details of these Hamiltonians are provided in Appendix C. The QWC fragments are obtained by the largest-first (LF) heuristic [4] and the SI algorithm [9]. A comparison of the number of fragments in QWC LF and QWC SI shows the slight advantage of QWC LF (Table I). However, the number of measurements is almost a factor of 2 lower for large Hamiltonians when fragments are defined by QWC SI (Table II). This shows a general trend that the number of fragments does not always correlate with the number of measurements. Also, this illustrates the advantage of the SI algorithm for the qubit term grouping; therefore, for the FC method, we use the SI algorithm as well.

All fermionic-algebra methods approximate the twoelectron integral tensor with a finite accuracy, which we judiciously choose to be $10^{-5}$ in the 1 -norm of the difference between all $g_{p q, r s}$ before the symmetrized algebra generator products and their restored values. For the SVD-factorization approach, the singular values arranged in descending order and their eigenvectors are used to reconstruct the $g_{p q, r s}$ matrix until the 1-norm threshold is satisfied.

For the FRO-based algorithms, it is found that real rotation generators in $\hat{U}_{\alpha}$ and real $\lambda^{(2, \alpha)}$ are sufficient for decomposition of the two-electron part of the electronic Hamiltonian. Additional simplification comes from the electron-spin symmetry in $g_{p q, r s}$, which allows us to manipulate with orbitals $(N / 2)$ rather than spin orbitals $(N)$. Therefore, we use the unitary operators $\hat{U}_{\alpha}$ generated by exponentiation of $\mathfrak{s o}(N / 2)$ instead of $\mathfrak{u}(N)$ and our $\hat{U}_{\alpha}$ operators are in the $\operatorname{Spin}(N / 2)$ subgroup of the original $U(N)$. As discussed in Ref. $10, \hat{U}_{\alpha}$ can be efficiently implemented $\left(N^{2} / 4-N / 2\right.$ two-qubit gates and a gate depth of exactly $N$ ) on a quantum computer with a limited connectivity. Limiting $\lambda^{(2, \alpha)}$ to real entries and accounting for its symmetric property $\left(\lambda_{t u}^{(2, \alpha)}=\lambda_{u t}^{(2, \alpha)}\right)$, the FRO procedures have $N^{2} / 4$ parameters in total for each fragment. For all molecules but $\mathrm{H}_{2}$, parameters $w$ [see Eq. (36)] and $\mu$ are set to 0.5 and 30 ; for $\mathrm{H}_{2}, w=0.5$ and $\mu=1$. To obtain fragment variance estimates for the VGFRO optimization, we use Hartree-Fock wave functions.

As expected, the QWC method results in one of the highest numbers of measurable groups and one of the highest numbers of required measurements. The FRO method, without any concern about fragment variances, provides the minimum number of fragments (Table I). However, more measurable fragments does not necessarily mean more measurements. As shown in Tables I and II, the GFRO and VGFRO methods partition the Hamiltonians into considerably more measurable parts than SVD and FRO, yet they improve on SVD consistently by $10 \%-30 \%$ in the number of measurements. This confirms the efficacy of the heuristic that groups operators with large coefficients in the same measurable fragment.

Interestingly, for the smaller molecules, FC SI outperforms the fermionic-algebra methods. This suggests that the CSA decomposition based on commutativity between Pauli products in qubit algebra is better at collecting large coefficients in few groups. However, since the numbers of measurable fragments in FC SI and SVD correspondingly scale with $O\left(N^{3}\right)$ and $O\left(N^{2}\right)[8,10]$, we expect that as the sizes of the systems grows, the fermionic-algebra methods that have more flexible measurable parts will better capture most coefficients in the Hamiltonians with fewer fragments and thus result in lower numbers of measurements, due to the nature of Eq. (34). The results for $N_{2}$ confirm that the

TABLE I. The number of measurable groups provided by different methods for Hamiltonians of several molecular systems [the number of spin orbitals $(N)$ and the total number of Pauli products in the qubit form (Total)]: qubit-algebra methods based on qubit-wise and full commutativity (QWC and FC), fermionic-algebra methods based on the SVD factorization (SVD) [10], full-rank optimization (FRO), greedy FRO (GFRO), and variance-estimate greedy FRO (VGFRO). The norm-1 accuracy for the fermionic-algebra methods is $10^{-5}$.

\begin{tabular}{|c|c|c|c|c|c|c|c|c|c|}
\hline Systems & $N$ & Total & QWC LF & QWC SI & FC SI & SVD & FRO & GFRO & VGFRO \\
\hline $\mathrm{H}_{2}$ & 4 & 15 & 3 & 3 & 2 & 4 & 3 & 3 & 4 \\
\hline $\mathrm{LiH}$ & 12 & 631 & 142 & 155 & 42 & 22 & 9 & 78 & 97 \\
\hline $\mathrm{BeH}_{2}$ & 14 & 666 & 172 & 183 & 36 & 29 & 13 & 118 & 129 \\
\hline $\mathrm{H}_{2} \mathrm{O}$ & 14 & 1086 & 313 & 334 & 50 & 29 & 11 & 119 & 148 \\
\hline $\mathrm{NH}_{3}$ & 16 & 3609 & 1272 & 1359 & 122 & 37 & 13 & 187 & 208 \\
\hline $\mathrm{N}_{2}$ & 20 & 2951 & 1177 & 1254 & 74 & 52 & 20 & 351 & 349 \\
\hline
\end{tabular}


TABLE II. The number of spin orbitals $(N)$ and the resulting number of measurements $\left(\epsilon^{2} K\right)$ required to estimate the energy expectation up to a standard deviation $\epsilon$. The fragment variances are calculated using the exact ground states of the Hamiltonians of the systems.

\begin{tabular}{lcccccccc}
\hline \hline Systems & $N$ & QWC LF & QWC SI & FC SI & SVD & FRO & GFRO & VGFRO \\
\hline $\mathrm{H}_{2}$ & 4 & 0.136 & 0.136 & 0.136 & 0.136 & 0.123 & 0.136 & 0.136 \\
$\mathrm{LiH}$ & 12 & 5.84 & 2.09 & 0.882 & 3.16 & 28.5 & 2.71 & 2.26 \\
$\mathrm{BeH}_{2}$ & 14 & 14.3 & 6.34 & 1.11 & 1.86 & 24.6 & 1.47 & 0.851 \\
$\mathrm{H}_{2} \mathrm{O}$ & 14 & 116 & 48.6 & 7.59 & 58.5 & 389 & 49.4 & 46.2 \\
$\mathrm{NH}_{3}$ & 16 & 352 & 97.0 & 18.8 & 58.1 & 471 & 47.0 & 42.2 \\
$\mathrm{~N}_{2}$ & 20 & 445 & 193 & 8.83 & 10.5 & 441 & 8.62 & 5.27 \\
\hline \hline
\end{tabular}

advantage of FC SI over SVD is shrinking and that the GFRO and VGFRO outperform FC SI.

\section{CONCLUSIONS}

In this work, we provide a unifying framework for many recently suggested approaches to efficient partitioning of quantum operators into measurable fragments. The framework is based on identifying a Lie algebra that is used for the operator expression (embedding), analysis of the corresponding Lie-group action, and the Cartan subalgebra of the Lie algebra. The latter encodes the measurable fragments, since it involves mutually commuting terms.

To obtain measurable fragments, we suggest two types of unitary transformation. First, there are the unitaries that are elements of the Lie group corresponding to the Lie algebra [Eq. (7)]. These unitaries have the advantage of conserving the degree of the Lie-algebra polynomials in the operator expression. Second, there are the unitaries that preserve the number of terms [Eq. (18)]. These unitaries use associative multiplication properties of algebraic operators and for qubit algebras correspond to the Clifford group. An intuitive rule for selection between these choices of unitaries is the degree of the Lie-algebraic operator polynomial expression: the Lie-group unitaries are more efficient for lower degrees and the unitaries that conserve the number of terms are more useful for higher degrees.

The ability to embed a single operator in multiple Lie algebras opens directions for a further search for efficient partitioning schemes. Here, we mainly focus on embedding of the electronic Hamiltonian in fermionic $[\mathfrak{u}(N)]$ and qubit $\left[\mathfrak{s u}\left(2^{N}\right)\right]$ Lie algebras. While the qubit embedding provides a more efficient scheme for electronic Hamiltonians of fewer orbitals than those of fermionic embedding, this trend can change as the size of the system grows.

To minimize the total number of measurements, it is not enough to reduce the number of measurable fragments because of the possible increase of the fragment variances. We find that grouping algorithms that employ greedy techniques is advantageous for lowering of the overall variance of the expectation-value estimator. This advantage can be attributed to lowering the total variance in cases when fragment variances are distributed nonuniformly. In addition, the fermionic CSA decomposition allows one to optimize partitioning by using fragment-variance estimates and to lower the overall variance in the VGFRO further than in the greedy approach (the GFRO).

\section{ACKNOWLEDGMENTS}

A.F.I. is grateful to Nicholas Rubin and William J. Huggins for providing details on performance of the algorithm from Ref. [10] and acknowledges financial support from the Google Quantum Research Program, Early Researcher Award and the Natural Sciences and Engineering Research Council of Canada.

\section{APPENDIX A: VARIOUS EMBEDDINGS OF THE ELECTRONIC HAMILTONIAN}

The electronic Hamiltonian can be written as

$$
\hat{H}_{e}=\sum_{p q} h_{p q} \hat{E}_{p}^{q}+\sum_{p q r s} g_{p q, r s} \hat{E}_{p}^{q} \hat{E}_{r}^{s}
$$

where the $h_{p q}$ and $g_{p q, r s}$ are one- and two-electronic integrals that are real constants [35] and the $\hat{E}_{p}^{q}=\hat{a}_{p}^{\dagger} \hat{a}_{q}$ are elements of the $\mathfrak{g l}(N)$ Lie algebra [23]

$$
\left[\hat{E}_{p}^{q}, \hat{E}_{r}^{s}\right]=\hat{E}_{p}^{s} \delta_{q r}-\hat{E}_{r}^{q} \delta_{p s}
$$

The $\hat{a}_{p}^{\dagger}, \hat{a}_{q}$ operators are regular fermionic creation and annihilation operators, where $p$ and $q$ run over $N$ spin orbitals. The CSA for $\mathfrak{g l}(N)$ consists of $N$ operators $\hat{E}_{p}^{p}$. A more common second quantized form of $\hat{H}_{e}$,

$$
\hat{H}_{e}=\sum_{p q} \tilde{h}_{p q} \hat{a}_{p}^{\dagger} \hat{a}_{q}+\sum_{p q r s} \tilde{g}_{p q, r s} \hat{a}_{p}^{\dagger} \hat{a}_{q}^{\dagger} \hat{a}_{r} \hat{a}_{s}
$$

can be considered as a different embedding (here, $\tilde{h}_{p q}$ and $\tilde{g}_{p q, r s}$ are real constants). To close the Lie algebra containing $\hat{a}_{p}^{\dagger}$ and $\hat{a}_{q}$ operators, one also needs to add products $\hat{a}_{p}^{\dagger} \hat{a}_{q}, \hat{a}_{p}^{\dagger} \hat{a}_{q}^{\dagger}$, and $\hat{a}_{p} \hat{a}_{q}$, which makes up the $\mathfrak{s o}(2 N+1)$ Lie algebra [36].

Another class of embeddings can be obtained by mapping fermionic operators to qubits using the Jordan-Wigner 
(JW), Bravyi-Kitaev (BK), or similar fermionic-qubit mappings [37-41]. Here, we use the JW mapping as the simplest for illustrative purposes:

$$
\begin{aligned}
& \hat{a}_{p}=\left(\hat{x}_{p}-i \hat{y}_{p}\right) \otimes \hat{z}_{p-1} \otimes \hat{z}_{p-2} \cdots \otimes \hat{z}_{1}, \\
& \hat{a}_{p}^{\dagger}=\left(\hat{x}_{p}+i \hat{y}_{p}\right) \otimes \hat{z}_{p-1} \otimes \hat{z}_{p-2} \cdots \otimes \hat{z}_{1} .
\end{aligned}
$$

This mapping produces

$$
\hat{H}_{q}=\sum_{k} c_{k} \hat{P}_{k}
$$

where the $c_{k}$ are numerical constants and the $\hat{P}_{k}$ are defined in Eq. (1). We can consider the $\hat{P}_{k}$ as elements of the UEA, where the Lie algebra is a direct sum of the $N \mathfrak{s u}(2)$ : $\mathcal{S}=\mathfrak{s u}(2) \oplus \cdots \oplus \mathfrak{s u}(2)$ and $\mathbb{K}=\mathbb{R} . \mathcal{S}$ is a semisimple Lie algebra with $3 N$ generators $\left(\hat{x}_{k}, \hat{y}_{k}\right.$, and $\left.\hat{z}_{k}\right)$.

Alternatively, instead of the UEA one can consider its isomorphic algebra, $\mathfrak{s u}\left(2^{N}\right)$, with $4^{N}-1$ generators given by the $\hat{P}_{k}$ operators. Accounting for time-reversal symmetry of the electronic Hamiltonian makes all the $c_{k}$ constants real and all the $\hat{P}_{k}$ to contain only products of an even number of $\hat{y}_{k}$ operators; hence, $\hat{H}_{q}$ is an element of the $\mathfrak{s o}\left(2^{N}\right)$ subalgebra of $\mathfrak{s u}\left(2^{N}\right)$. Thus, the qubit embedding $\hat{H}_{q}$ can be seen either as an element of the UEA of $\mathcal{S}$ or as an element of the $\mathfrak{s o}\left(2^{N}\right)$ Lie algebra.

There are $3^{N}$ CSAs for $\mathcal{S}$, which are based on selecting a particular Pauli operator $(\hat{x}, \hat{y}$, or $\hat{z})$ for each qubit and thus contain $N$ elements each; for example, a directly measurable CSA is $\left\{\hat{z}_{k}\right\}_{k=1}^{N}$.

Out of the total $2^{N}\left(2^{N}-1\right) / 2$ generators of $\mathfrak{s o}\left(2^{N}\right)$, one can select exponentially many CSAs. They can be obtained by constructing UEAs from CSAs of $\mathcal{S}$ and removing those that contain an odd number of $\hat{y}$ operators to maintain a real character. This generates exponentially large CSAs with $2^{N}-1$ commuting elements, for example, for two qubits, a directly measurable CSA has basis elements $\hat{z_{1}}, \hat{z_{2}}$, and $\hat{z}_{1} \hat{z}_{2}$.

\section{APPENDIX B: SINGULAR-VALUE DECOMPOSITION FOR THE TWO-ELECTRON HAMILTONIAN}

Here, we show how SVD can provide a heuristic factorization of the two-electron Hamiltonian part

$$
\hat{H}_{e}^{(2)}=\sum_{p q r s}^{N} g_{p q, r s} \hat{E}_{p}^{q} \hat{E}_{r}^{s}
$$

SVD for $g_{p q, r s}$ gives

$$
g_{p q, r s}=\sum_{k}^{N^{2}} U_{p q, k} \Lambda_{k} U_{k, r s}^{\dagger}
$$

$$
=\sum_{k}^{N^{2}} U_{p q, k} \Lambda_{k}^{1 / 2}\left[U_{r s, k} \Lambda_{k}^{1 / 2}\right]^{\dagger}
$$

$$
=\sum_{k}^{N^{2}} L_{p q}^{(k)}\left(L_{r s}^{(k)}\right)^{\dagger}
$$

where the $\Lambda_{k}$ are singular values. Each matrix $L_{p q}^{(k)}$ can be diagonalized as $\sum_{r}\left(U^{(k)}\right)_{p r}^{\dagger} \omega_{r}^{(k)} U_{r q}^{(k)}$ for fixed $k$. This allows us to write the two-electron tensor as

$$
g_{p q, r s}=\sum_{k}^{N^{2}} \sum_{t u}^{N}\left(U^{(k)}\right)_{p t}^{\dagger} \omega_{t}^{(k)} U_{t q}^{(k)}\left(U^{(k)}\right)_{r u}^{\dagger} \omega_{u}^{(k)} U_{u s}^{(k)} .
$$

The entire $\hat{H}_{e}^{(2)}$ then can be written as

$$
\hat{H}_{e}^{(2)}=\sum_{p q r s t u, k}\left[\left(U^{(k)}\right)_{p t}^{\dagger} \hat{E}_{p}^{q} U_{t q}^{(k)}\right] \omega_{t}^{(k)} \omega_{u}^{(k)}\left[\left(U^{(k)}\right)_{r u}^{\dagger} \hat{E}_{r}^{s} U_{u s}^{(k)}\right] .
$$

It is easy to show that the operators in the square brackets,

$$
\hat{B}_{t}^{(k)}=\sum_{p q}\left(U^{(k)}\right)_{p t}^{\dagger} \hat{E}_{p}^{q} U_{t q}^{(k)}
$$

are from the CSA and are equivalent to $\hat{E}_{t}^{t}$.

To show the commutativity of $\hat{B}_{t}^{(k)}$, let us consider their commutators for a fixed $k$ :

$$
\left[\hat{B}_{t}^{(k)}, \hat{B}_{u}^{(k)}\right]=\sum_{p q, r s}\left(U^{(k)}\right)_{p t}^{\dagger} U_{t q}^{(k)}\left(U^{(k)}\right)_{r u}^{\dagger} U_{u s}^{(k)}\left[\hat{E}_{p}^{q}, \hat{E}_{r}^{s}\right] .
$$

$\left[\hat{E}_{p}^{q}, \hat{E}_{r}^{s}\right]=\hat{E}_{p}^{s} \delta_{q r}-\hat{E}_{r}^{q} \delta_{p s}$; therefore, we can consider two parts

$$
\hat{E}_{p}^{s}: \sum_{p q, r s}\left(U^{(k)}\right)_{p t}^{\dagger} U_{t q}^{(k)}\left(U^{(k)}\right)_{r u}^{\dagger} U_{u s}^{(k)} \hat{E}_{p}^{s} \delta_{q r}
$$

$$
=\sum_{p q s}\left(U^{(k)}\right)_{p t}^{\dagger} U_{t q}^{(k)}\left(U^{(k)}\right)_{q u}^{\dagger} U_{u s}^{(k)} \hat{E}_{p}^{s}
$$

$$
=\sum_{p s}\left(U^{(k)}\right)_{p t}^{\dagger} \delta_{t u} U_{u s}^{(k)} \hat{E}_{p}^{s}
$$

$$
=\sum_{p s}\left(U^{(k)}\right)_{p t}^{\dagger} U_{t s}^{(k)} \hat{E}_{p}^{s}=\hat{B}_{t}^{(k)}
$$

Analogously, for the $\hat{E}_{r}^{s}$ part, we obtain the same result but with the minus sign. Thus the commutator is zero. 
Therefore, one can measure each $k$ part of the twoelectronic Hamiltonian:

$$
\hat{H}_{e}^{(2)}=\sum_{k}^{N^{2}} \sum_{t u}^{N} \omega_{t}^{(k)} \omega_{u}^{(k)} \hat{B}_{t}^{(k)} \hat{B}_{u}^{(k)} .
$$

Considering the norm of $\Omega_{t u}^{(k)}=\omega_{t}^{(k)} \omega_{u}^{(k)}$ for each $k$, one can reduce the number of measurable sets if this norm is below a certain threshold.

\section{APPENDIX C: DETAILS OF HAMILTONIANS}

The Hamiltonians are generated using the STO-3G basis and the $\mathrm{BK}$ transformation. The nuclear geometries for the Hamiltonians are $\mathrm{R}(\mathrm{H}-\mathrm{H})=1 \AA\left(\mathrm{H}_{2}\right), \mathrm{R}(\mathrm{Li}-\mathrm{H})=1 \AA$ $(\mathrm{LiH})$, and $\mathrm{R}(\mathrm{Be}-\mathrm{H})=1 \AA$, with collinear atomic arrangement $\left(\mathrm{BeH}_{2}\right), \mathrm{R}(\mathrm{O}-\mathrm{H})=1 \AA$, with $\angle \mathrm{HOH}=107.6^{\circ}$ $\left(\mathrm{H}_{2} \mathrm{O}\right), \mathrm{R}(\mathrm{N}-\mathrm{H})=1 \AA$, with $\angle \mathrm{HNH}=107^{\circ}\left(\mathrm{NH}_{3}\right)$, and $\mathrm{R}(\mathrm{N}-\mathrm{N})=1 \AA\left(\mathrm{N}_{2}\right)$.

[1] A. Peruzzo, J. McClean, P. Shadbolt, M.-H. Yung, X.-Q. Zhou, P. J. Love, A. Aspuru-Guzik, and J. L. O'Brien, A variational eigenvalue solver on a photonic quantum processor, Nat. Commun. 5, 4213 (2014).

[2] J. I. Cirac and P. Zoller, Goals and opportunities in quantum simulation, Nat. Phys. 8, 264 (2012).

[3] J. Argüello-Luengo, A. González-Tudela, T. Shi, P. Zoller, and J. I. Cirac, arXiv.org arXiv:1807.09228 (2018).

[4] V. Verteletskyi, T.-C. Yen, and A. F. Izmaylov, Measurement optimization in the variational quantum eigensolver using a minimum clique cover, J. Chem. Phys. 152, 124114 (2020).

[5] A. Kandala, A. Mezzacapo, K. Temme, M. Takita, M. Brink, J. M. Chow, and J. M. Gambetta, Hardware-efficient variational quantum eigensolver for small molecules and quantum magnets, Nature 549, 242 (2017).

[6] T.-C. Yen, V. Verteletskyi, and A. F. Izmaylov, Measuring all compatible operators in one series of single-qubit measurements using unitary transformations, J. Chem. Theory Comput. 16, 2400 (2020).

[7] A. Jena, S. Genin, and M. Mosca, arXiv.org, arXiv:1907.07859 (2019).

[8] P. Gokhale, O. Angiuli, Y. Ding, K. Gui, T. Tomesh, M. Suchara, M. Martonosi, and F. T. Chong, arXiv.org, arXiv:1907.13623 (2019).

[9] O. Crawford, B. van Straaten, D. Wang, T. Parks, E. Campbell, and S. Brierley, arXiv e-prints arXiv:1908.06942 [quant-ph] (2019).

[10] W. J. Huggins, J. McClean, N. Rubin, Z. Jiang, N. Wiebe, K. B. Whaley, and R. Babbush, arXiv e-prints arXiv:1907.13117 [quant-ph] (2019).

[11] X. Bonet-Monroig, R. Babbush, and T. E. O'Brien, arXiv.org arXiv:1908.05628v1 (2019).

[12] A. F. Izmaylov, T.-C. Yen, R. A. Lang, and V. Verteletskyi, Unitary partitioning approach to the measurement problem in the variational quantum eigensolver method, J. Chem. Theory Comput. 16, 190 (2020).
[13] R. Prevedel, P. Walther, F. Tiefenbacher, P. Böhi, R. Kaltenbaek, T. Jennewein, and A. Zeilinger, High-speed linear optics quantum computing using active feed-forward, Nature 445, 65 (2007).

[14] J. M. Pino, J. M. Dreiling, C. Figgatt, J. P. Gaebler, S. A. Moses, M. S. Allman, C. H. Baldwin, M. Foss-Feig, D. Hayes, K. Mayer, C. Ryan-Anderson, and B. Neyenhuis, arXiv.org arXiv:2003.01293v2 (2020).

[15] A. F. Izmaylov, T. C. Yen, and I. G. Ryabinkin, Revising the measurement process in the variational quantum eigensolver: Is it possible to reduce the number of separately measured operators?, Chem. Sci. 10, 3746 (2019).

[16] S. Aaronson, Shadow tomography of quantum states, arXiv:1711.01053 [quant-ph] (2018).

[17] H.-Y. Huang, R. Kueng, and J. Preskill, Predicting many properties of a quantum system from very few measurements, Nat. Phys. 16, 1050 (2020).

[18] A. Zhao, N. C. Rubin, and A. Miyake, Fermionic Partial Tomography via Classical Shadows, Phys. Rev. Lett. 127, 110504 (2021).

[19] C. Hadfield, Adaptive Pauli shadows for energy estimation, arXiv:2105.12207 [quant-ph] (2021).

[20] C. Hadfield, S. Bravyi, R. Raymond, and A. Mezzacapo, Measurements of quantum Hamiltonians with locallybiased classical shadows, arXiv:2006.15788 [quant-ph] (2020).

[21] H.-Y. Huang, R. Kueng, and J. Preskill, Efficient Estimation of Pauli Observables by Derandomization, Phys. Rev. Lett. 127, 030503 (2021).

[22] T.-C. Yen, A. Ganeshram, and A. F. Izmaylov, Deterministic improvements of quantum measurements with non-local transformations, overlapping frames, greedy grouping, and covariance estimates (to be published).

[23] R. Gilmore, Lie Groups, Physics, and Geometry: An Introduction for Physicists, Engineers and Chemists (Cambridge University Press, Cambridge, 2008).

[24] A. O. Barut and R. Raczka, in Theory of Group Representations and Applications (Polish Scientific Publisher, Warszawa, Poland, 1980), p. 17.

[25] All commuting elements of the semisimple Lie algebra must be ad-diagonalizable.

[26] B. C. Hall, in Lie Groups, Lie Algebras, and Representations: An Elementary Introduction (Springer, Switzerland, 2015), p. 314.

[27] A. O. Barut and R. Raczka, Theory of Group Representations and Applications (Polish Scientific Publisher, Warszawa, Poland, 1980).

[28] W. H. Press, B. P. Flannery, S. A. Teukolsky, and W. T. Vetterling, Numerical Recipes (Cambridge University Press, Cambridge, 1992).

[29] I. G. Ryabinkin, S. N. Genin, and A. F. Izmaylov, Relation between fermionic and qubit mean fields in the electronic structure problem, J. Chem. Phys. 149, 214105 (2018).

[30] A Clifford group consists of unitaries that transform a single Pauli product into another Pauli product.

[31] F. L. Hitchcock, The expression of a tensor or a polyadic as a sum of products, J. Math. Phys. 6, 164 (1927).

[32] T. G. Kolda and B. W. Bader, Tensor decompositions and applications, SIAM Rev. Soc. Ind. Appl. Math. 51, 455 (2009). 
[33] $\operatorname{Spin}(K)$ is a Lie group that originates from exponentiation of $\mathfrak{s o}(K)$ generators.

[34] N. C. Rubin, R. Babbush, and J. McClean, Application of fermionic marginal constraints to hybrid quantum algorithms, New J. Phys. 20, 053020 (2018).

[35] T. Helgaker, P. Jørgensen, and J. Olsen, Molecular Electronic-Structure Theory (John Wiley \& Sons, Ltd, Chichester, UK, 2000).

[36] H. Fukutome, The group theoretical structure of fermion many-body systems arising from the canonical anticommutation relation. I: Lie algebras of fermion operators and exact generator coordinate representations of state vectors, Prog. Theor. Phys. 65, 809 (1981).

[37] S. B. Bravyi and A. Y. Kitaev, Fermionic quantum computation, Ann. Phys. 298, 210 (2002).
[38] J. T. Seeley, M. J. Richard, and P. J. Love, The Bravyi-Kitaev transformation for quantum computation of electronic structure, J. Chem. Phys. 137, 224109 (2012).

[39] A. Tranter, S. Sofia, J. Seeley, M. Kaicher, J. McClean, R. Babbush, P. V. Coveney, F. Mintert, F. Wilhelm, and P. J. Love, The Bravyi-Kitaev transformation: Properties and applications, Int. J. Quantum Chem. 115, 1431 (2015).

[40] K. Setia and J. D. Whitfield, Bravyi-Kitaev superfast simulation of electronic structure on a quantum computer, J. Chem. Phys. 148, 164104 (2018).

[41] V. Havlíček, M. Troyer, and J. D. Whitfield, Operator locality in the quantum simulation of fermionic models, Phys. Rev. A 95, 032332 (2017). 